\title{
Trihelix transcription factor GT-4 mediates salt tolerance via interaction with TEM2 in Arabidopsis
}

\author{
Xiao-Hong Wang ${ }^{\dagger}$, Qing-Tian Li ${ }^{\dagger}$, Hao-Wei Chen, Wan-Ke Zhang, Biao Ma, Shou-Yi Chen ${ }^{*}$ and Jin-Song Zhang ${ }^{*}$
}

\begin{abstract}
Background: Trihelix transcription factor family is plant-specific and plays important roles in developmental processes. However, their function in abiotic stress response is largely unclear.

Results: We studied one member GT-4 from Arabidopsis in relation to salt stress response. GT-4 expression is induced by salt stress and GT-4 protein is localized in nucleus and cytoplasm. GT-4 acts as a transcriptional activator and its C-terminal end is the activation domain. The protein can bind to the cis-elements GT-3 box, GT-3b box and MRE4. GT-4 confers enhanced salt tolerance in Arabidopsis likely through direct binding to the promoter and activation of Cor15A, in addition to possible regulation of other relevant genes. The gt-4 mutant shows salt sensitivity. TEM2, a member of AP2/ERF family was identified to interact with GT-4 in yeast two-hybrid, BiFC and Co-IP assays. Loss-of-function of TEM2 exerts no significant difference on salt tolerance or Cor15A expression in Arabidopsis. However, double mutant gt-4/tem2 shows greater sensitivity to salt stress and lower transcript level of Cor15A than gt-4 single mutant. GT-4 plus TEM2 can synergistically increase the promoter activity of Cor15A.
\end{abstract}

Conclusions: GT-4 interacts with TEM2 and then co-regulates the salt responsive gene Cor15A to improve salt stress tolerance.

Keywords: Salt stress, Trihelix transcription factor, GT-4, TEM2

\section{Background}

Plant growth, development and productivity are greatly affected by adverse environmental conditions such as drought, cold and high salinity. A plenty of genes have been reported to respond to these abiotic stresses. Among them, transcription factor genes are important for adaptation to these stresses. Several classes of transcription factors have been found to play important roles in plant stress tolerance through binding of cis-acting elements in the promoter region of stress-responsive genes [1-9].

The trihelix transcription factor family is defined according to the highly conserved trihelix domain which specifically binds to the GT-elements $[10,11]$. The trihelix domain has similarities to the individual repeats of the MYB family from which the trihelix may have been derived [12]. Compared with other transcription factors, e.g. MYB, AP2/EREBP, bHLH, NAC and WRKY families with more than 100 members in Arabidopsis, trihelix family

\footnotetext{
* Correspondence: sychen@genetics.ac.cn; jszhang@genetics.ac.cn ${ }^{\dagger}$ Equal contributors

State Key Lab of Plant Genomics, Institute of Genetics and Developmental Biology, Chinese Academy of Sciences, Beijing 100101, China
}

is relatively small [13]. Until now, there are 30 members in Arabidopsis and 31 members in rice, and the members in Arabidopsis can be grouped into five classes, namely GT-1, GT-2, SH4, GT $\gamma$ and SIP1. Each class is named after the relevant founding member [14,15].

Since members of trihelix family specifically bind with GT elements, these proteins are also named as GT factor. The first trihelix transcription factor was identified in pea (Pisum sativum) and named GT-1 factor. It binds specifically to a light-responsive GT element, named Box II/GT1box (5'-GTGTGGTTAATATG-3'), in the pea $r b c S-3 A$ gene promoter. The core sequence 5'-GGTTAA-3' is sufficient for light induction [16,17]. Later, GT-elements were found in many promoters of genes, some of which were not responsive for light [11]. For instance, a GT element named Site1, found in the ribosomal protein gene rps1 promoter, represses transcription in non-photosynthetic tissues or cells [18,19]. Box II-related/GT-1 like element in the promoter region of $\mathrm{Pr}-1 \mathrm{~A}$ gene from tobacco is likely responsive to pathogen infection [20]. Soybean SCaM-4 gene with GT-1 element in the promoter region interacts with GT1-like 
factor and can be induced by pathogen attack and $\mathrm{NaCl}$ treatment [21].

Current information suggests that trihelix transcription factors not only regulate light-responsive genes [17,22-24] but also play important roles in the regulation of developmental processes involving flowers, trichomes, stomata, embryos and seeds [14,25-28] and in responses to biotic and abiotic stresses [21,29-33].

Arabidopsis PETAL LOSS (PTL), which belongs to the GT-2 group, was the first trihelix gene identified associating with a morphogenetic function. PTL regulates petal and sepal growth, and sepal fusion [25,28,34]. Rice Shattering 1(SHA1) gene encoding a SH4-type factor plays an important role in activation of cell separation, and a mutation in the trihelix domain results in the elimination of seed shattering in cultivated rice [26]. More recently, ASIL1, belonging to a new subfamily of trihelix family, has been found to function as a negative regulator of a large subset of Arabidopsis embryonic and seed maturation genes in Arabidopsis seedlings [14]. The role for GT-3b in responding to salt and pathogen stress is also identified in Arabidopsis. GT-3b expression is rapidly induced by $\mathrm{NaCl}$ and Pseudo-monas syringae infection and GT-3b binds to a GT-like element (GAAAAA) in the promoter of a calmodulin gene (SCaM-4) [21]. We demonstrates that overexpression of GmGT-2A or GmGT-2B from soybean enhanced tolerance to salt, drought and freezing stresses in transgenic Arabidopsis plants [29].

Although the roles of the GT factors are gradually disclosed, the regulatory functions of this kind of transcription factors in abiotic stress response remains largely unknown. In the present study, we find that expression of GT-4 is induced by high salinity. Mutation of the gene causes sensitivity to salt stress and transgenic plants overexpressing GT-4 exhibits salt tolerance compared to Col-0. We further identified a B3 and AP2/ERF domain-containing protein (TEM2) that interacted with GT-4. Loss function of TEM2 in gt-4 mutant affected plant performance under salt stress. The downstream gene Cor15A was co-regulated by GT-4 and TEM2. The GT-4 may associate with TEM2 to co-activate Cor15A for salt stress tolerance.

\section{Results}

\section{GT-4 expression and protein subcellular localization}

There are 26 members of GT family in Arabidopsis and we examined expressions of all of these genes in response to various stresses [35]. One of the stress-responsive genes, named GT-4 (At3g25990), was further analyzed. GT-4 encoded a protein of 372 amino acids and the protein had a trihelix DNA binding domain in the $\mathrm{N}$-terminus and a variable $\mathrm{C}$-terminus. Arabidopsis seedlings were treated with salt stress and the expression of GT-4 was clearly induced by high salinity (Figure 1a). The expression of
GT-4 was also examined in different organs of Arabidopsis plant. Figure 1b showed that GT-4 expressed ubiquitously and was abundant in rosette leaves and pods but less expressed in roots (Figure 1b).

We determined the subcellular localization of GT-4. GT-4 was fused to the GFP gene in a transient expression vector. The fusion gene and GFP control driven by CaMV $35 \mathrm{~S}$ promoter were transformed into Arabidopsis protoplasts to observe the localization of GT-4. The green fluorescence from GT-4-GFP fusion protein was localized in both nuclear and cytoplasm regions (Figure 1c). The GFP control protein was similarly localized.

\section{Transcriptional activation ability of GT-4}

GT factors usually function as transcription factors and we measured the transcriptional activation ability of GT-4 by using a dual-luciferase reporter (DLR) assay system in Arabidopsis protoplasts [1]. Different regions of GT-4 were also examined for the activation. The domains of GT-4 were analyzed using SMART program and GT-4 protein was divided into N-terminal (amino acid No. 1 to 113, 1/113) and C-terminal (amino acid No. 114 to 372,114/372). The full length, N-terminal (1/113) and C-terminal (114/372) coding regions of GT-4 protein were fused to the GAL4 DNA-binding domain to generate pBD-GT-4, pBD-GT-4-N and pBD-GT-4-C effector plasmids respectively (Figure 1d). The fusion genes were driven by the $35 \mathrm{~S}$ promoter plus a translational enhancer $\Omega$. The firefly luciferase gene $(L U C)$ driven by a mini-35S (TATA box) promoter with five copies of the GAL4 binding element was used as a reporter (Figure 1d), and the Renilla luciferase gene driven by the Arabidopsis Ubiquitin3 promoter was used as an internal control. VP16, a herpes simplex virus (HSV)-encoded transcriptional activator protein was used as a positive control. The GAL4 DNA-binding domain in the fusion proteins binds to the GAL4 binding element upstream of the reporter $L U C$ gene, and the activation domain in the tested proteins activates $L U C$ gene transcription. Compared with the GAL4-DBD negative control, full length GT-4 and C-terminal region (114/373) of GT-4 could activate the reporter gene, whereas the $\mathrm{N}$-terminal region (1/113) of GT-4 didn't have the ability to activate reporter gene expression (Figure 1e). The results indicated that GT-4 and its C-terminal domain possess transcriptional activation ability.

\section{DNA-binding ability of GT-4}

GT proteins specifically bind to GT elements, and the elements are highly degenerated. GT-1 and GT-3 proteins with one trihelix DNA-binding domain specially bind to Box II core sequence (5'-GTGTGGTTAATATG-3') and the 5'-GTTAC-3' sequence respectively. GT-2 protein with two trihelix DNA-binding domains can bind to GT-2 box 
(a)

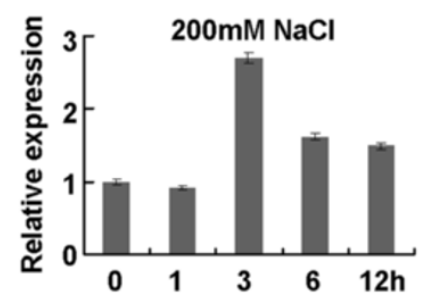

(b)

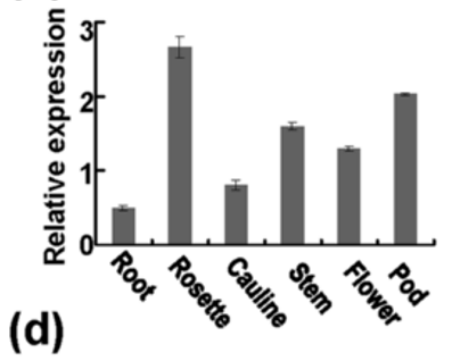

(c)

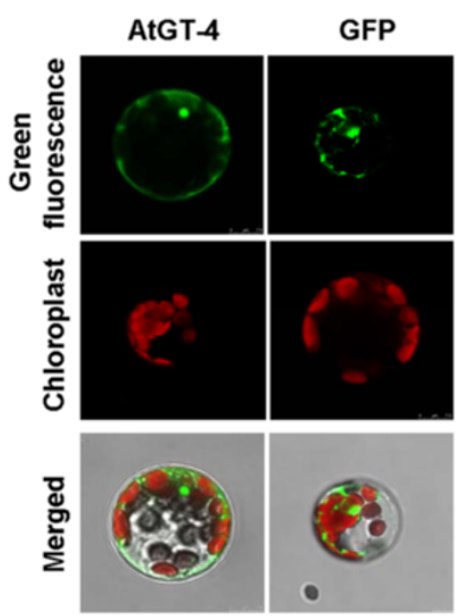

\section{Effectors}

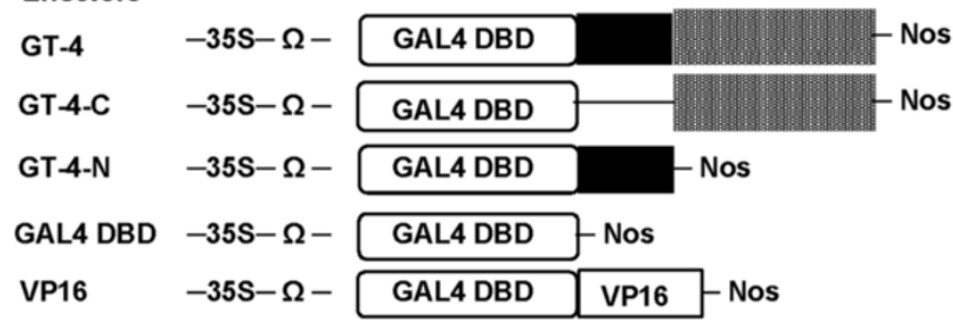

(e)

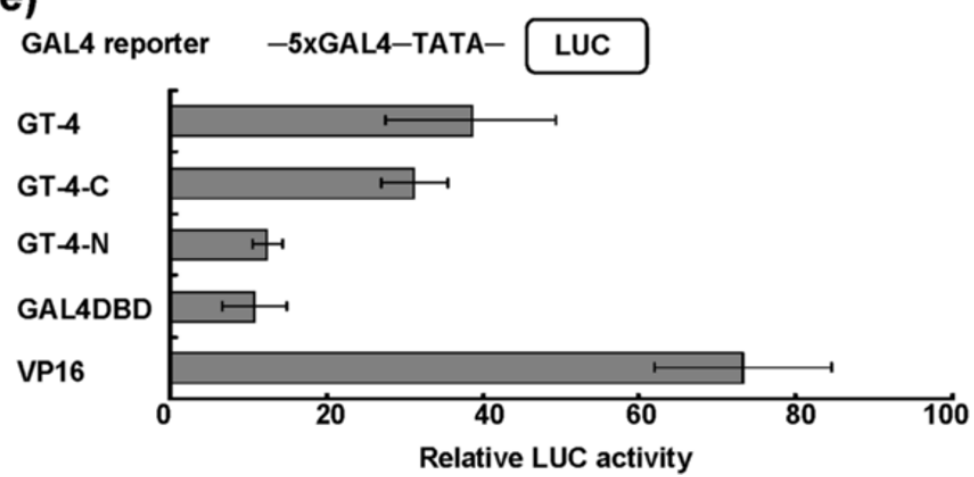

Figure 1 GT-4 gene expression and protein localization and transcriptional activation ability. (a) GT-4 expression levels in response to salt stress. Bars indicate SD $(n=3)$. (b) GT-4 expression in various organs of Arabidopsis plants. Bars indicate SD $(n=3)$. (c) Subcellular localization of GT-4 protein in Arabidopsis protoplasts. (d) Effector constructs used in the Arabidopsis protoplast transient assay. Each effector contained a GAL4 DNA-binding domain (GAL4DBD). The GAL4DBD effector was used as a negative control, and effector VP16, was used as a positive control. Full length GT-4, GT-4-N (1-113) and GT-4-C (114-372) was fused with the GAL4DBD and expression was driven by the 355 promoter plus the translation enhancer $\Omega$ sequence. (e) Transcriptional activation ability of GT-4, GT-4-N and GT-4-C as revealed by relative LUC activity of the reporter. The effectors and the GAL4-LUC reporter were co-transfected. Bars indicate SD $(n=4)$.

(5'-GCGGTAATTAA-3') and GT-3 box (5-GAGGTAAAT CCGCGA-3) sequences [17,36,37]. There are reports that trihelix proteins resemble those of MYB proteins [12]. Several known GT elements and MYB protein binding elements were selected as binding elements (P1 to P8) to identify the DNA-binding ability of the present GT-4 by
EMSA (Figure 2, upper panel). GT-4 formed a complex with P1 (GT-3 box), P2 (GT-3b box) and P7 (MRE4), and the intensity of the retarded bands were dramatically reduced when non-labeled competitors were included (Figure 2, lower panel), indicating that GT-4 specifically binds to these elements. It should be noted that the GT-4 


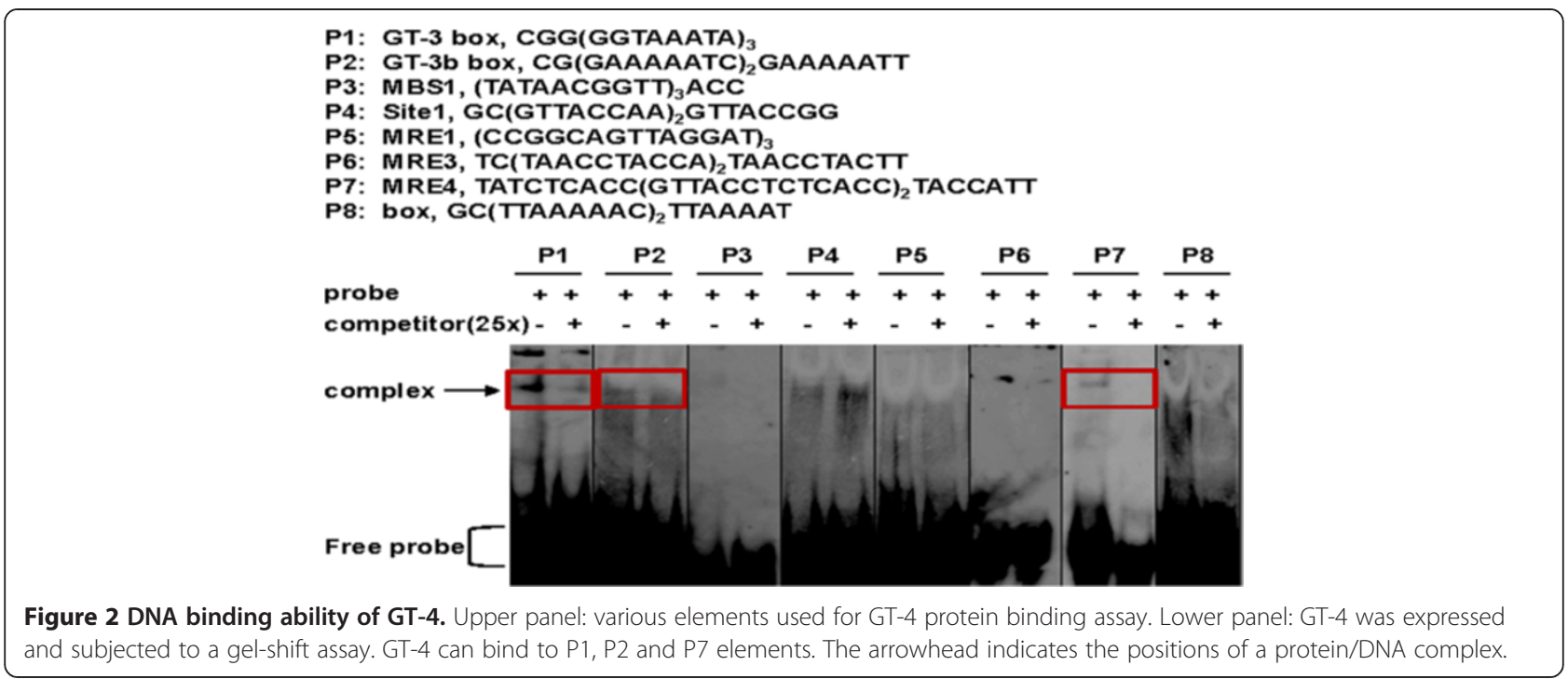

may also bind to the P3 (MBS1) and P8 (box) probes since addition of the competitors seemed to reduce the band intensities slightly (Figure 2).

\section{Performance of mutant gt-4 and transgenic Arabidopsis plants overexpressing GT-4 under salt stress}

To elucidate the biological function of GT-4, one T-DNA insertion mutant was identified and designated as gt-4 (SALK_095404). The T-DNA insertion was located in the first exon of GT-4 (Figure 3a) and was confirmed by PCR (Figure 3b). No full-length transcript of GT-4 was detected in the mutant $g t-4$ by RT-PCR (Figure 3b), suggesting that $g t-4$ was loss-of-function mutant. Transgenic Arabidopsis plants overexpressing GT-4 driven by the CaMV $35 \mathrm{~S}$ promoter were generated. At least 60 transgenic lines were obtained, and two independent homozygous lines (GT-4-OE) L47 and L54 with relatively high expression of GT-4 (Figure 3c) were further investigated. Since GT-4 was responsive to salt stress, we tested if it is involved in regulation of stress tolerance. Under normal growth condition, mutant gt-4, GT-4-OE and Col-0 plants showed normal growth (Figure 3d). All plants (7-day old) were transferred to soils in pots saturated with $\mathrm{NaCl}$ solutions and grew for 5 weeks (Figure 3d). The gt-4 mutants were more sensitive to salt and transgenic plants were more tolerant to salinity than Col- 0 as can be seen from both the growth performance and the survival rate under $125 \mathrm{mM}$ and $150 \mathrm{mM} \mathrm{NaCl}$ treatments (Figure 3d,e). These results indicate that GT-4 plays a positive role in the regulation of plant tolerance to salt stress.

\section{GT-4 regulates expressions of Cor15A}

Expression of stress-related genes was examined in mutant gt-4 and GT-4- overexpressing plants grown under normal condition by quantitative PCR. Compared with that in
Col-0, the expressions of Cor15A (At2g42540) was enhanced in GT-4-OE lines but decreased in $g t-4$ plants (Figure 4a). The result implies that GT-4 may confer stress tolerance through activation of Cor15A. We determined whether GT-4 regulates $\operatorname{Cor} 15 A$ by direct binding to its promoter region and the EMSA was performed. Since GT protein can bind to GT-3b box, GT-4 may bind to the same element in the promoter region of downstream genes. A 60 bp DNA fragment from the Cor15A promoter was identified to contain the GT-3b box. GT- 4 was found to specifically bind to this sequence from Cor $15 A$ promoter (Figure $4 \mathrm{~b}$ ). These results indicate that GT-4 most likely activates Cor $15 A$ expression through direct binding to the GT-3b box in its promoter region.

\section{GT-4 interacts with TEM2, a protein with B3 and AP2/ERF domain}

Transcription factors were reported to interact with the same family proteins or other transcription factors [38]. The proteins interacted with GT-4 were identified by using a yeast two-hybrid assay system. GT-4-coding sequence was cloned into pGBKT7 vector and the recombinant BD-GT-4 protein was used as a bait to screen an Arabidopsis prey cDNA library. Among four unique genes encoding putative GT-4-interacting proteins, a cDNA encoding a transcription factor TEM2 (At1g68840) containing an AP2/ERF domain was selected for further investigation. To clarify the interaction, the coding sequence of TEM2 was fused to the 3'-end of the GAL4 activation domain $(\mathrm{AD})$ coding region to generate pGADT7TEM2. Combinations corresponding to AD-TEM2 mating with BD-GT-4 showed a clear positive interaction on the QDO/X/A plate (Figure 5b). We also investigated the interacting domain of GT-4 with TEM2, and found that 
(a)

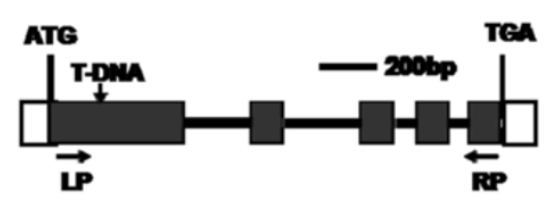

(b)

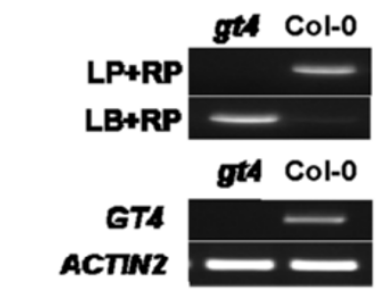

(c)



(d)
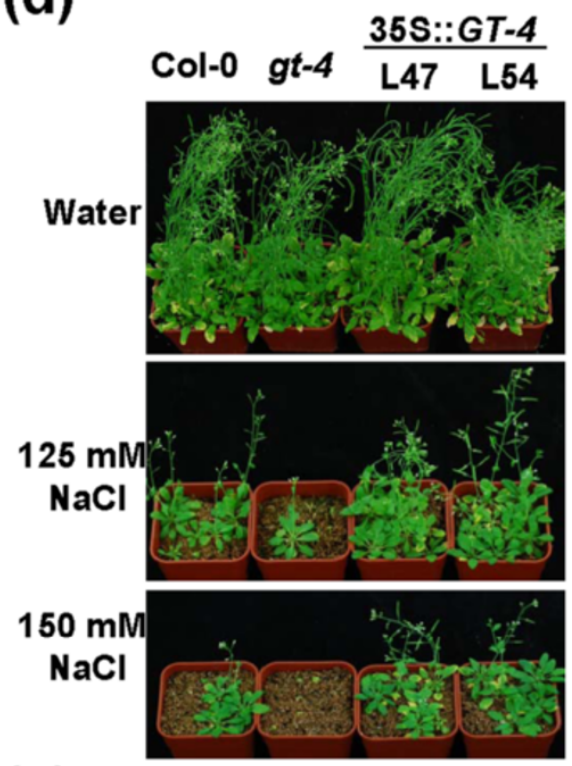

(e)

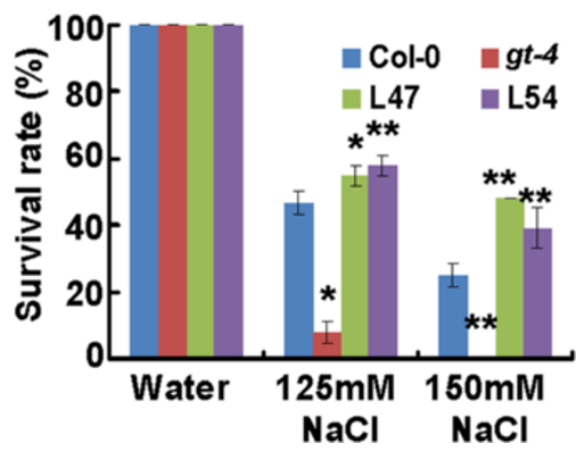

Figure 3 Identification of $\boldsymbol{g t}$ - $\mathbf{4}$ mutant and GT-4-overexpressing lines and performance of these plants under salt stress. (a) T-DNA insertion site in GT-4 in the gt-4 mutant. The filled black boxes represent ORFs, while the lines between the boxes represent introns. LP and RP are primers used for PCR analysis. (b) RT-PCR analysis of the GT-4 transcript levels in seedlings of Col-0 and mutant lines. The Actin2 gene was used as an internal control. (c) GT-4 transcripts in Col-0 and GT-4-over-expression plants by qRT-PCR analysis. Bars indicate SD ( $n=3$ ). (d) Performance of various plants under salt stress. Seven-day-old plants were transferred to $\mathrm{NaCl}$-containing pot and grew for 5 weeks. (e) Survival rates of plants after salt treatments. Bars indicate SD $(n=4)$. Each replicate uses 16 plants. Asterisks indicate a significant difference compared to the corresponding Col-0 (*P $<0.05$ and $\left.{ }^{* * P}<0.01\right)$.

the C-terminal but not the N-terminal end of GT-4 interacted with TEM2 (Figure 5b). Furthermore, GT-4 can form homo-dimer itself (Figure 5a). BD-GT-4, BD-GT-4 N, BD-GT-4C, AD-GT-4 and AD-TEM2 fusion plasmids were respectively transfected into the yeast cells as negative controls and the corresponding proteins showed no auto-activation or DNA-binding abilities (see Additional file 1).

To visualize the co-localization of GT-4 and TEM2, the full-length coding sequences (CDS) of GT-4 and TEM2 without stop codon were cloned into pGWB405 and pGWB454 vectors with C-terminal sGFP and mRFP fusion respectively. Confocal analysis revealed that the GT-4 and TEM2 co-localized in the nucleus (Figure 5c).
A bimolecular fluorescence complementation (BiFC) system was used to further characterize the interaction between GT-4 and TEM2 in planta. GT-4 tagged with C-terminal (YC) and TEM2 tagged with N-terminal of yellow fluorescent protein $(\mathrm{YN})$ were transiently co-expressed in Arabidopsis protoplasts, and the yellow fluorescence was detected in the nuclei of Arabidopsis protoplasts (Figure $5 \mathrm{~d}$ ). However, YC-GT-4 and YN or YN-TEM2 and YC did not exhibit any fluorescence (see Additional file 2). These results suggest that GT-4 can interact with TEM2 in the nuclei of plant cells.

To further confirm the interaction between GT-4 and TEM2 in vivo, a Co-immunoprecipitation (Co-IP) assay was performed. Two constructs 35S-Myc-TEM2 


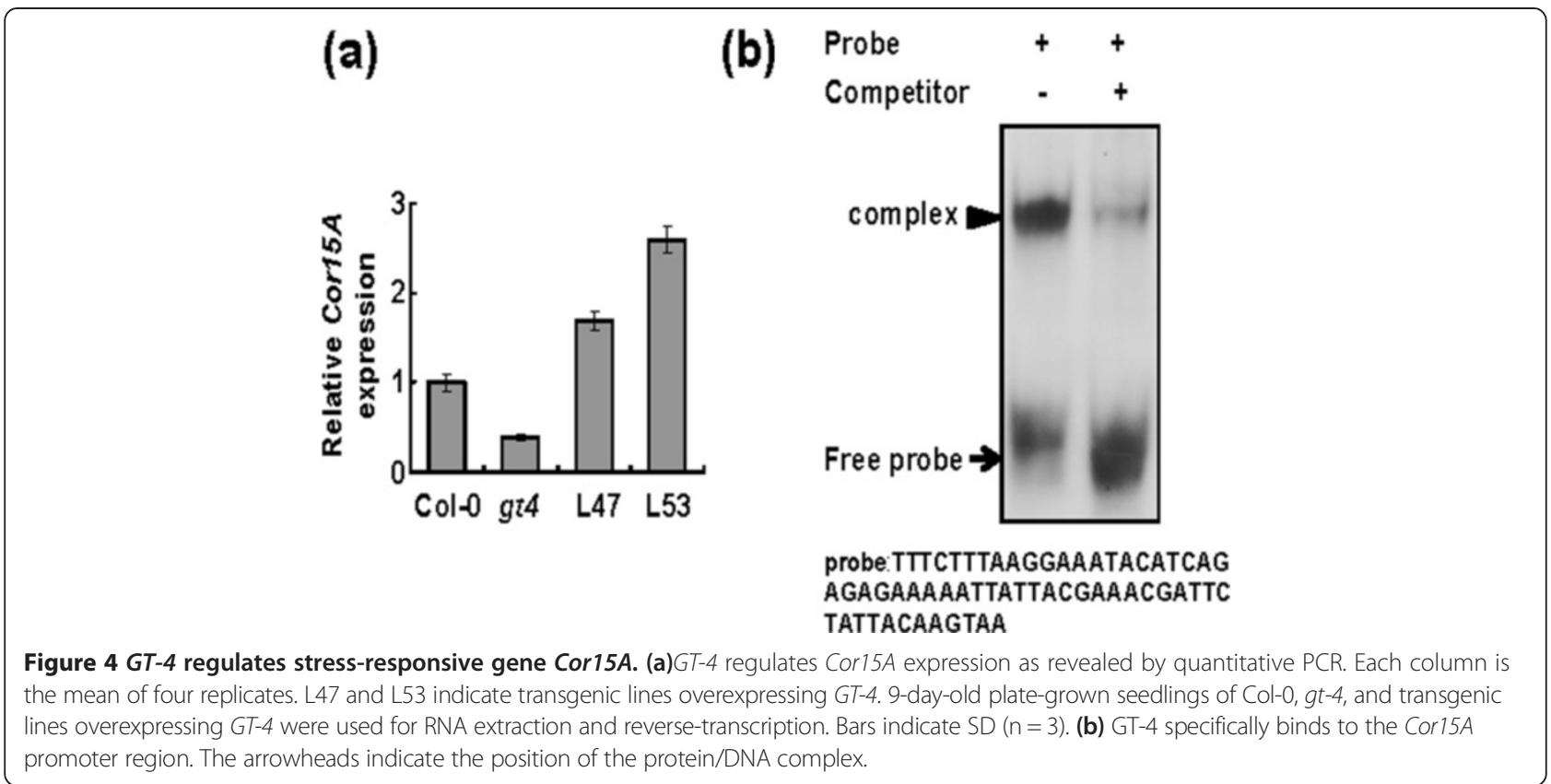

and 35S-GT-4-Flag were produced using the Gateway system. Argo-infiltration procedure was then performed with minor modifications. Proteins were isolated from tobacco leaves expressing 35S-Myc-TEM2 and 35S-GT-4-Flag, and then incubated with anti-c-Myc agarose beads. The proteins were then eluted and followed by western blotting analysis with anti-Flag or anti-Myc antibodies. Figure 5e showed that GT-4 interacted with TEM2 in an in vivo assay.

\section{The GT-4 and TEM2 affect salt stress response via co-regulation of Cor15A}

Previous reports have found that TEM2 acts as a transcriptional repressor and is regulated by light and the circadian clock. Over-expression of TEM2 severely delayed the flowering time of Arabidopsis [39], yet the role of TEM2 in salt stress response is unclear. We thus analyzed the function of TEM2 in salt stress responses. The expression of TEM2 was suppressed after initiation of salinity and then recovered to normal level subsequently (Figure 6f). One T-DNA insertion mutant was identified and designated as tem2 (SALK_070847). The insertion was located in the only exon (Figure 7a) and was confirmed by PCR (Figure 6b). No TEM2 expression was detected in the mutant by qRT-PCR (Figure 6c), suggesting that tem 2 is a loss-of-function mutant.

To further analyze the mechanism of GT-4 and TEM2 in salt stress response, $g t-4$ mutant was crossed with tem 2 mutant to obtain double mutant $g t-4 /$ tem 2 . We then examined the salt stress response of Col-0, gt-4, tem 2 and gt-4/tem2. The 10-day-old Col-0 and mutant plants were transferred into soil saturated with $150 \mathrm{mM}$ $\mathrm{NaCl}$ and maintained for a period of $10 \mathrm{~d}$. No obvious difference was observed between Col-0 and tem2, whereas a remarkably low number of $g t-4 /$ tem 2 plants were found to survive the exposure to salinity in comparison with the control and $g t-4$ plants (Figure 6d,e). Under normal condition, no obvious difference was observed between Col-0 and various mutants (Figures 6d).

Real-time quantitative PCR analysis was performed to examine the expressions of Cor15A in Col-0, gt-4, tem2, gt-4/tem2 mutant plants and GT-4-OE line 47 (L47) under salt stress. The Col-0 and tem 2 displayed similar levels of $\operatorname{Cor} 15 A$ expression under salt stress. The $g l-4$ mutant showed an approximate two-fold decrease of Cor $15 A$ expression compared to the Col-0 level throughout the treatments. In the double mutant gt-4/tem2, more severe decrease of Cor15A expression was observed than those in the other plants tested. Cor15A transcript level in L47 was always higher than that in Col-0 (Figures 7a).

We further confirmed qPCR results by an in vivo transient luciferase expression system. The Cor15A promoter was fused to the LUC reporter, and the resulting construct was co-transformed into tobacco leaves with pGWB454-TEM2 and/or pGWB454-GT-4. We found that GT-4 could activate the Cor $15 A$ promoter activity whereas TEM2 didn't significantly affect this activity. The activation was stronger when these two proteins were co-expressed (Figures 7b). These results indicate that GT-4/TEM2 complex are transcriptional activator of Cor15A expression to regulate salt response.

\section{Discussion}

Previously, we have found that soybean trihelix transcription factors $G m G T-2 A$ and $G m G T-2 B$ improve plant 
(a)

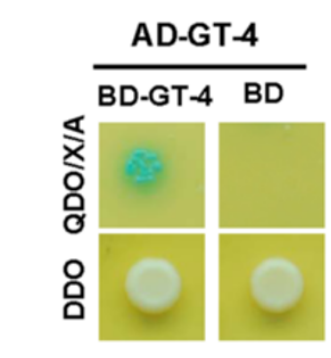

(c)

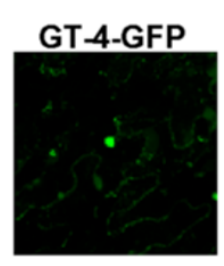

Merged

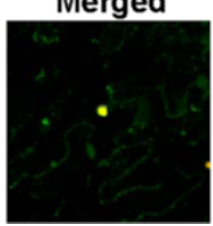

(b)
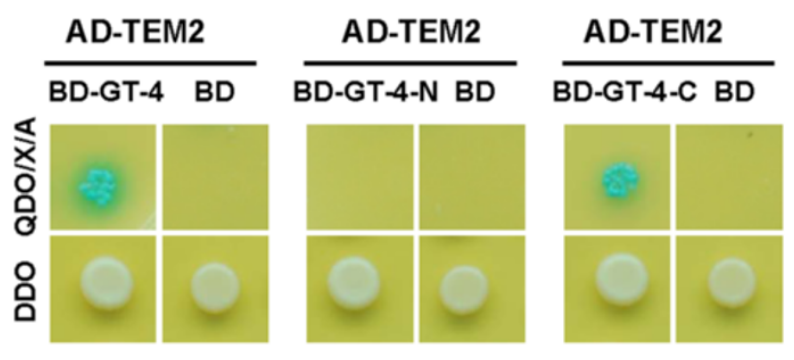

(d)
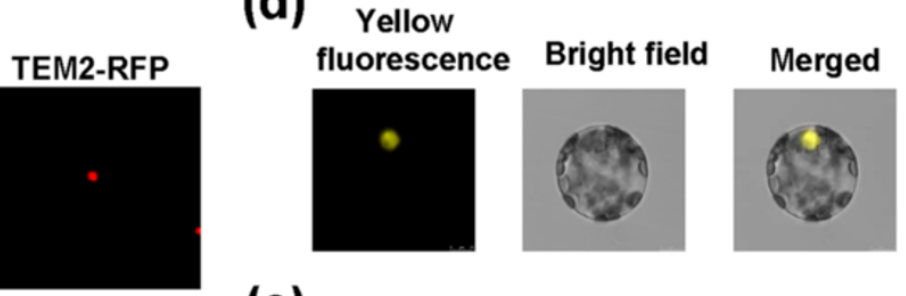

Bright field



(e)

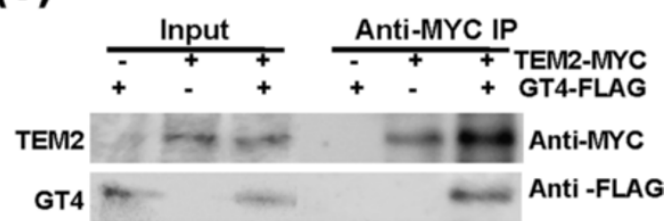

Figure 5 GT-4 interacts with TEM2 in vivo and in vitro. (a) Dimerization of Arabidopsis GT-4 in yeast two-hybrid assay. The yeast cells harboring the plasmid combinations were grown on DDO plates (SD medium without Leu and Trp) and QDO/X/A plates (SD/-Ade/-His/-Leu/-Trp medium containing $40 \mu \mathrm{g} \mathrm{mL}-1 \mathrm{X}-\mathrm{a}-\mathrm{Gal}$ and $125 \mathrm{ng} \mathrm{mL}-1$ Aureobasidin A) for $3 \mathrm{~d}$. The cells generating blue color indicate positive interactions between the two proteins. (b) GT-4 and C-terminal of GT-4 interact with TEM2 in yeast two hybrid assay. (c) GT-4 and TEM2 co-localized in the nucleus. Tobacco leaves were co-infiltrated with 35S:GFP-GT-4 and 35S:RFP-TEM2. Signals were observed directly under a confocal microscope after 3 days. (d) Bimolecular fluorescence complementation (BiFC) assay. The fusion constructs were co-transformed into Arabidopsis protoplasts and the cells were observed $16 \mathrm{~h}$ later under a confocal microscope. YFP fluorescence was excited at a wavelength of $488 \mathrm{~nm}$. (e) Co-IP assay. Nuclei were isolated from tobacco leaves expressing 35S:GT-4-FLAG and 35S:TEM2-MYC. Then proteins were extracted and incubated with anti-c-Myc agarose beads. The proteins were then eluted and followed by western blotting analysis with anti-flag or anti-Myc antibodies.

tolerance to abiotic stresses in transgenic Arabidopsis [29]. GT2L was reported to interact with calmodulin and respond to salt stress and regulate expression of $R D 29 \mathrm{~A}$ and $E R D 10$ [40]. In the present study, GT-4 was identified to be stress-responsive and conferred salt tolerance in transgenic Arabidopsis plants through regulation of downstream gene Cor15A. The gt-4 mutant was sensitive to salt stress as revealed by phenotypic change. GT-4 binds to the GT-3b box in the Cor15A promoter and activates its expressions. A B3 and AP2/ERF domain containing protein TEM2 was identified to interact with GT-4 and jointly regulate salt tolerance.

Various binding elements of trihelix proteins have been identified. Previous study showed that the GT-4 recombinant protein binds to the GT1-box, GT2-box and GT3-box $[15,41]$. However, the positions of the indicated GT-4 protein/DNA complex are very low and near to the free probe regions [41]. The GT-4 gene is also induced by light [41]. In our present study, we found that GT-4 not only binds to the GT-elements, such as GT-3 box and
GT-3b box, but also bind to the MYB protein binding element MRE4 with weaker affinity (Figure 2). In addition, the position of our protein/DNA complex is high compared to that from Murata et al. [41]. This discrepancy is likely due to the fact that we used probe elements with two or three repeats whereas Murata et al. (2002) used only one repeat [41]. Moreover, the higher positions of the protein/DNA complex in Figure 3 may also be due to the dimerization of the GT-4 protein since it has the ability to form dimers (Figure 6a).

Transcription factors have either transcriptional activation or suppression activities. Hao et al. (2011) reported that GmNAC11 had transcriptional activation activity whereas GmNAC20 had suppression activity, and have identified a NARD domain in plant NAC-type transcription factors for suppression of transcriptional activation [1]. Cotton TCP protein GhTCP14 contains transcriptional activation activity, however, it can directly down-regulate the expression of PIN2 possibly by its interaction with other proteins [42]. A rice GT-2 protein has been found to function as a 


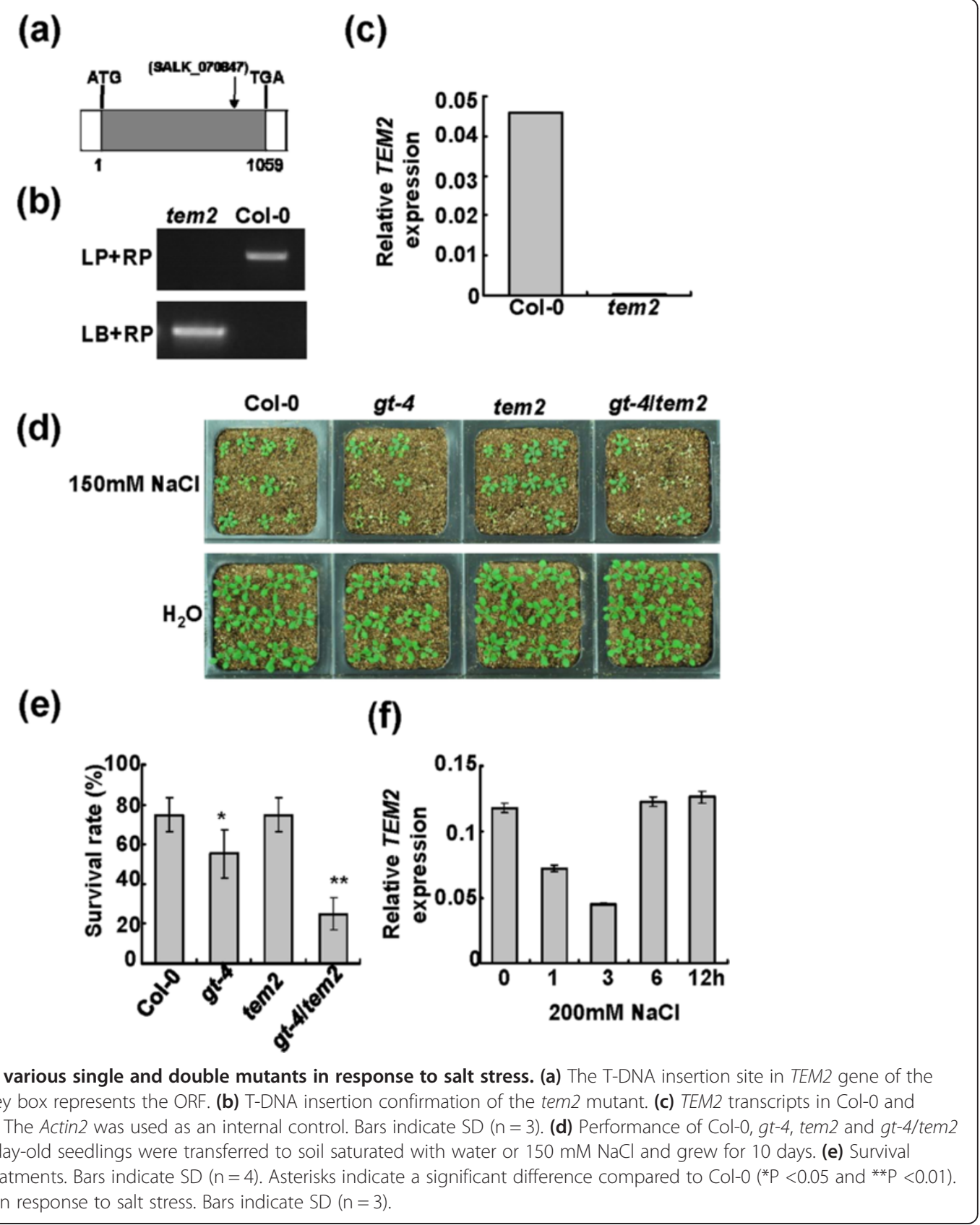

transcriptional activator. However, the activation domain was not identified [43]. Arabidopsis GT-1 also has transactivation function in both yeast and plant cells [44]. GTL1 functions as a transrepressor for SDD1 gene in stomatal development in Arabidopsis [31]. Presently, we find that GT-4 exhibited transcriptional activation activity in protoplast assay and the activation domain may be located in the C-terminal end (amino acids 114-372) of GT-4 (Figure 1b). The N-terminal end (amino acids 1-113) containing the trihelix domain may function as the DNA-binding domain. The minimal domain for transcriptional activation needs further study.
GT-4 may enhance stress tolerance by regulating downstream stress-responsive gene $\operatorname{Cor} 15 A$ through direct binding to the GT-3b box in the Cor $15 A$ promoter (Figure 4). The Cor15A protein shows homology to the LEA protein family. The Cor15A may protect lactate dehydrogenase from aggregation during stress $[45,46]$. Overexpression of the Cor $15 \mathrm{~A}$ gene in Arabidopsis leads to accumulation of the protein in chloroplast stroma and confers freezing tolerance [47]. Transgenic Arabidopsis overexpressing Cor15A exhibited greater $\mathrm{NaCl}$ tolerance than the wild-type in saline soil [48]. Therefore, increased expression of Cor15A in GT-4 overexpression 

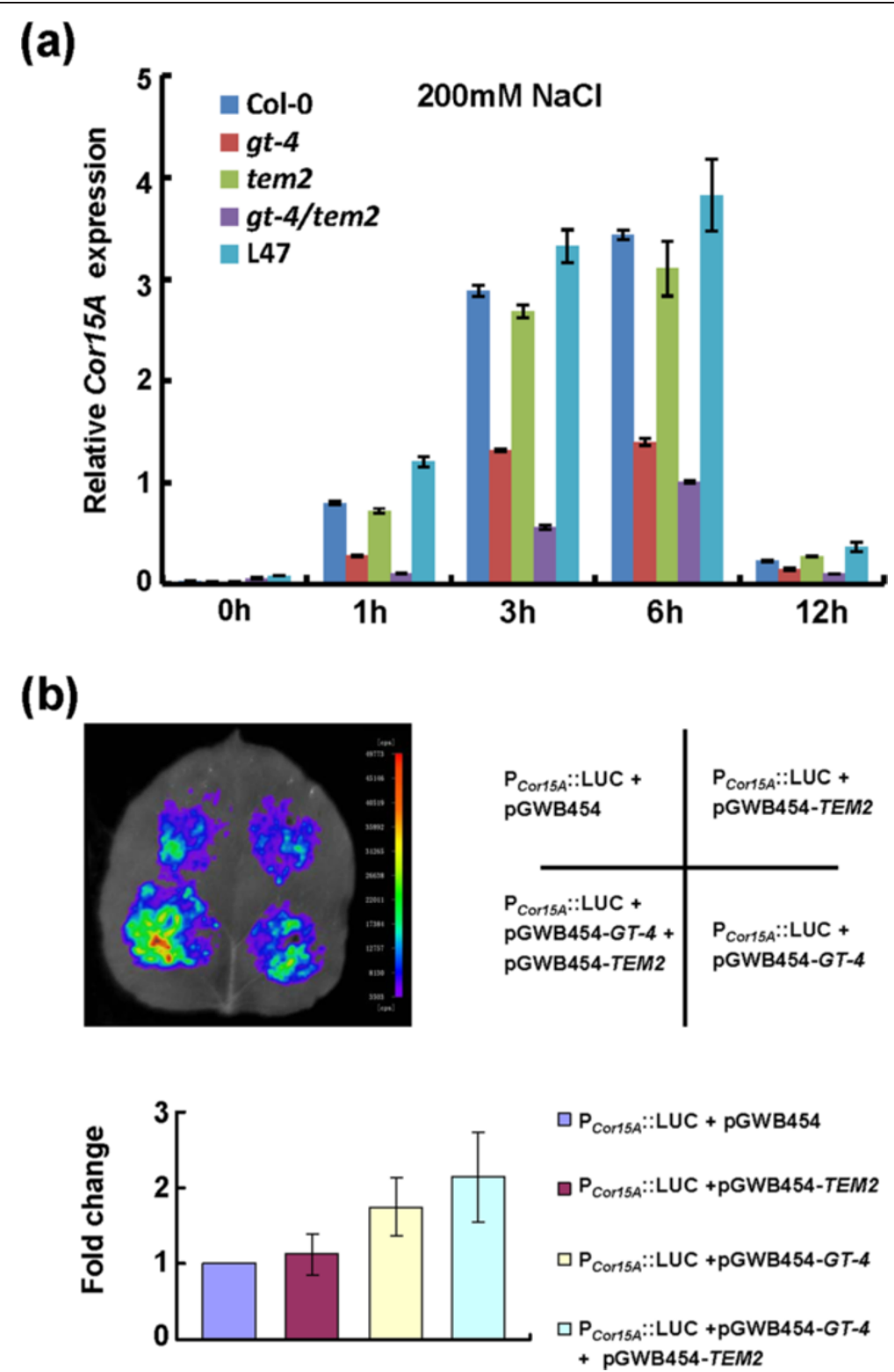

Figure 7 Interaction of GT-4 and TEM2 activates Cor15A expressions. (a) Cor15A transcripts in Col-0, mutant plants and L47 with $200 \mathrm{mM}$ $\mathrm{NaCl}$ treatment for 1 h, $3 \mathrm{~h}, 6 \mathrm{~h}$ and $12 \mathrm{~h}$. Bars indicate SD $(\mathrm{n}=3)$. (b) GT-4 plus TEM2 activate Cor15A promoter activity in tobacco leaves. The $A$.

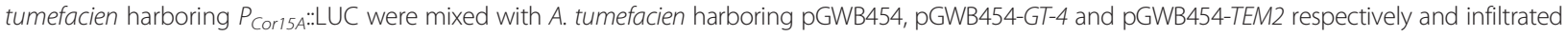
into tobacco leaves. LUC image was taken 2 days after infiltration. Fluorescence intensity was analyzed with IndiGo software (lower panel). Each column is the mean of more than 5 leaves. Bars indicate SD.

plants may provide protection by preventing damage of chloroplast membrane and enzymes from salt stress. It should be noted that in GT-4 overexpressing plants, the GT-4 expression is enhanced by around 15-fold while the Cor15A level is only enhanced by 2 -fold (Figure 4). This discrepancy may be due to that the GT-4 protein levels and/or its activities are not elevated in proportion. Alternatively, other relevant downstream genes may also be regulated for stress responses.
Most trihelix proteins have been identified to localize to the nucleus $[25,29,37,49,50]$. GT- 4 has been reported to localize to the nucleus of the onion epidermal cells [41]. However, in the present study, it should be mentioned that GT-4 not only localize in the nucleus but also in the cytoplasm (Figures 1e, 5c), suggesting that GT-4 may have other regulatory roles in the cytoplasm in addition to its function as a transcriptional activator in the nucleus and it needs to be further studied. 
Through yeast two-hybrid assay, BiFC and Co-IP, we find that GT-4 could interact with TEM2 and the interaction seems to be mediated by the $\mathrm{C}$-terminal of GT-4 (Figure 5a and b). TEM2 may enhance the activation activity of GT-4 via interacting with $\mathrm{C}$-terminus activation domain (Figure 5b, 7b). It has been reported that Arabidopsis GT-3a and GT-3b could form homo or heterodimers, and the dimerization domain seemed to be located at the C-terminus [37]. Likewise, GT-4 can interact with itself that may function as homo-dimer to regulate gene expression. It is possible that GT-4 dimerization may facilitate the association of GT-4 with TEM2, and whether this is the case remains to be further studied. TEM2 is a member of RAVE subfamily of AP2-EREBP family and play an important role in flower development and mechanical stimuli response [39,51]. Members of AP2-EREBP family have been shown to be involved in enhancement of salt tolerance [52-54].

Cor15A expression increases in Arabidopsis in response to $\mathrm{NaCl}$ with transcript levels peaking at $6 \mathrm{~h}$ and returning to near basal levels within $12 \mathrm{~h}$. The expression levels of $\operatorname{Cor} 15 \mathrm{~A}$ roughly correlate with the survival rates of the double and single mutants after salt treatment (Figures 6e, 7a). The above results seem to be consistent with the ability of GT-4, TEM2 or their combination in activation of $\operatorname{Cor} 15 A$ promoter activities (Figure $7 \mathrm{~b}$ ).

The salt-inductions of Cor $15 A$ expression in tem2 mutant is largely unchanged, suggesting that there is no obvious correlation between Cor $15 \mathrm{~A}$ expression and TEM2 (Figure 7a). Additionally, overexpression of TEM2 exerts no influence on $\mathrm{P}_{\text {Cor15A }}:$ LUC (Figure $7 \mathrm{~b}$ ). However, the double mutant gt-4/tem 2 show a more severe reduction in Cor $15 \mathrm{~A}$ expression and salt tolerance than $g t-4$ single mutant (Figures 6d,e and 7a), indicating that, under high-salinity condition, activation of Cor $15 \mathrm{~A}$ and promotions of tolerance by elevated GT-4 expression partially depend on TEM2 function and TEM2 regulate salt stress response through interaction with GT-4 on the whole. It is possible that the interaction between GT-4 and TEM2 would enhance the transcriptional activation ability of GT-4 and then promote Cor15A expressions. This finding raises the possibility that GT-4 acts as a transcription activator in cooperation with the TEM2 under salinity condition and confers stress tolerance. It should be noted that, under normal condition, the Cor15A expression levels increase in the $g t-4 /$ tem2 mutant, probably reflecting a feedback mechanism for maintainment of the normal growth and development.

\section{Conclusions}

Collectively, we demonstrate that GT-4 has important roles in adaptation to salt stress through regulating Cor15A. TEM2, as a novel GT-4-interacting partner with B3 and AP2/ERF domains, also participates in salt stress responses via interaction with GT-4. Our results reveal mechanisms of GT-4 in salt stress tolerance and provide novel gene resources for crop improvement.

\section{Methods}

\section{Plant growth and treatments}

Arabidopsis seeds (Columbia ecotype, Col-0) were surfacesterilized, plated on 1/2 Murashige and Skoog (MS) medium, stratified at $4^{\circ} \mathrm{C}$ for 3-4 d and then germinated at $23^{\circ} \mathrm{C}$ under $16 \mathrm{~h}$ photoperiod. For salt stress, 6-day-old seedlings from Col-0, gt-4, tem 2 and transgenic lines overexpressing GT-4 were transferred into soil containing various concentrations of $\mathrm{NaCl}$. For each $\mathrm{NaCl}$ treatment, at least three replicates were performed.

\section{Identification of T-DNA insertion mutants}

T-DNA insertion mutant of $g t-4$ (SALK_095404) and tem2 (SALK_070847) was obtained from SALK database. The seed samples were sowed on 1/2MS medium. PCR screening for insertions was carried out using gene specific primer pairs. GT-4-LP is 5'-TGAGATCAATACCTTCAA CAGATG-3' and GT-4-RP is 5'- TTGTGTGCTGTTTGT TCGAAG- 3'; TEM2-LP is 5'-GTGTTGTTCCTCAGCC TAACG-3' and TEM2-RP is 5'- TTTCCACAAAACCATT GTTCC-3'. RT-PCR analysis of full length gene expression was used to evaluate the effect of insertion. The Actin 2 was amplified as a control.

\section{Generation of transgenic Arabidopsis plants}

The coding region of GT-4 was amplified from cDNA with primers containing BamHI/HindIII sites, and cloned into the pCAMBIA1302 vector. The gene was driven by the $35 \mathrm{~S}$ promoter. The forward primer is 5'CGGGATCCATGTTTGTTTCCGATAAC -3' and the reverse primer is 5'-CCCAAGCTTTCTCATTCCTCTG TATAAG-3'. The expression plasmid pCAMBIA-GT-4 was transfected into agrobacterium GV3101 and then transformed into Arabidopsis plants using floral dip method. T3 homozygous plants with higher level of transgene expression were used for further analysis.

\section{qRT-PCR analysis}

Total RNA from aerial parts of four-week-old plate-grown plants was used for reverse-transcription (RT) with MMLV reverse transcriptase according to the manufacture's protocol (Promega). Genes selected and corresponding primers were shown in Additional file 3. Real-time quantitative PCR was performed on Roche Light Cycler 480 using the SYBR green PCR kit (TOYOBO, Osaka, Japan) and PCR was conducted according to the following protocol: $15 \mathrm{~s}$ denaturation at $94^{\circ} \mathrm{C}, 15 \mathrm{~s}$ annealing at $57^{\circ} \mathrm{C}, 15 \mathrm{~s}$ elongation at $72^{\circ} \mathrm{C}$ in 40 cycles. Fluorescence was detected at $80^{\circ} \mathrm{C}$. Samples were analyzed in triplicate 
using independent cDNA samples and were quantified by the comparative cycle threshold method [55].

\section{Subcellular localization analysis}

The open reading frame (ORF) of GT-4 was amplified by RT-PCR with the specific primers 5'-CGGGATCCAT GTTTGTTTCCGATAAC-3' and 5'-CCCAAGCTTTCT CATTCCTCTGTATAAG-3'. The PCR products were digested with BamH I and HindIII, and fused to GFP in pBIN221. The fusion gene and the GFP control were driven by the $35 \mathrm{~S}$ promoter. The two constructs were transformed into Arabidopsis protoplasts with polyethylene glycol (PEG) treatment, and GFP signal was detected by Leica TCS SP5 microscope.

\section{Transcriptional activation assay in Arabidopsis protoplasts}

Full length and N- and C- terminal sequences of GT-4 were obtained by PCR. For full length sequence, the forward primer is 5'-CGGGATCCATGTTTGTTTCC GATAAC-3' and the reverse primer is 5'-GGGGTAC CCCTCTCATTCCTCTGTATAAG-3'; for N-terminal sequence, the forward primer is 5'- GCTCTAGAAT GTTTGTTTCCG ATAAC-3' and the reverse primer is 5'-GGGGTACCCTCTTTCAATA TGTTCCTC-3'; for the C-terminal sequence, the forward primer is 5'-GCTC TAGATTTAAGAAAGCTAAGCAAC-3' and the reverse primer is 5'-GGGGTACCTCATCTCATTCCTCTGTA TAAG-3'. The GAL4 DNA-binding domain (BD)-coding sequence was fused to the above genes and inserted into the pRT107 to generate effector plasmids pRTBD-GT-4. The fusion genes were under the control of $35 \mathrm{~S}$ promoter. The BD sequence was also fused to VP16 gene to generate positive control effector plasmid. The pRT107 containing the BD sequence was used as negative control. The reporter plasmid containing 5xUAS and $35 \mathrm{~S}$ promoter upstream of a reporter gene encoding a firefly luciferase (LUC) was used. The effector and reporter plasmids were co-transfected into Arabidopsis protoplasts and the relative LUC activity was determined based on previous descriptions [56]. The experiments have been repeated independently for three times and the results were consistent. Results from one experiment were presented.

\section{EMSA assay}

The GT-4 coding region was amplified and cloned into the BamHI/XhoI sites of the pGEX6p-1 vector containing a GST tag. The forward primer is 5'-CGGGATCCAT GTTTGTTTCCGATAACAAC-3' and the reverse primer is 5'- CCGCTCGAGTCTCATTCCTC TGTATAAGC-3'. The GST-GT-4 fusion protein was expressed in Escherichia coli (BL21) and purified using Glutathione Sepharose 4B (GE). Oligonucleotides and their reverse complementary oligonucleotides were synthesized, and the sequences are shown in Figure 3. Double-stranded DNA was obtained by heating oligo-nucleotides at $70^{\circ} \mathrm{C}$ for $5 \mathrm{~min}$, and annealing at room temperature in $50 \mathrm{mM} \mathrm{NaCl}$ solution. The gel-shift assay was performed as described previously [57] using digoxigenin-labeled probes. For the experiment in Figure 4b, the Arabidopsis Cor $15 \mathrm{~A}$ promoter sequence was used.

\section{Yeast two-hybrid interaction assay}

The cDNA library was constructed using a Make Your Own "Mate \& Plate" Library System (Clontech, Mountain View, CA, USA) according to the manufacturer's instructions. Briefly, total RNA was extracted from 2-week-old Arabidopsis seedlings air-dried for $3 \mathrm{~h}$ and $200 \mathrm{mM} \mathrm{NaCl}$ treated for $6 \mathrm{~h}$. Library cDNAs were integrated into the linearised pGADT7-Rec vector by co-transformation and recombination in yeast strain Y187. The coding sequence of GT-4 was amplified by PCR using gene-specific primers containing NdeI and EcoRI sites. The forward primer is 5'GGAATTCCATATGATGTTTGTTTCCGATAAC-3' and the reverse primer is 5- GGAATTC TCATCTCATTCCT CTGTATAAGCG-3, and fused to the coding sequence of DNA binding domain of GAL4 to construct plasmids pGBKT7-GT-4. A yeast strain Y2HGold transformed with pGBKT7-GT-4 was mixed with pre-transformed Y187 library and the mated strains were selected on high stringency medium (SD/-Ade/-His/-Leu/-Trp medium containing $40 \mu \mathrm{g} \mathrm{mL}-1 \mathrm{X}-\alpha-\mathrm{Gal}$ and $125 \mathrm{ng} \mathrm{mL}-1$ Aureobasidin A). Plasmid DNA was isolated from the positive clone and the cDNA fragment was sequenced.

\section{Co-localization analysis in Nicotiana benthamiana}

The full-length coding sequence (CDS) of GT-4 and TEM2 without stop codon were amplified and inserted into the Gateway entry vector and then cloned into pGWB405 and pGWB454 vectors with C-terminal sGFP and mRFP fusion. The argo-infiltration was performed as described below and a Leica TCS SP5 confocal laser scanning microscope was used for confocal assay. Wavelengths to visualize GFP and RFP were 500 to 540 and 600 to $640 \mathrm{~nm}$, respectively.

\section{Bimolecular fluorescence complementation (BiFC) assay in Arabidopsis protoplasts}

The ORFs of GT-4 and TEM2 were amplified by PCR using gene-specific primers and fused to the 5 ' end of $\mathrm{N}$-terminal or C-terminal of yellow fluorescent protein (YFP) coding region to form GT-4-YFPc and TEM2-YFPn. For the BiFC protein-protein interaction analysis, the above constructs were introduced into Arabidopsis protoplasts via PEG-mediated transformation, and the transgenic protoplasts were observed with a laser scanning confocal microscope (Leica, Germany). 


\section{Co-immunoprecipitation in Nicotiana benthamiana}

Two constructs pGWB411-10XMyc-GT-4 and pGWB421Flag-TEM2 were made using the Gateway system. Argo-infiltration procedure was performed according to Liu et al. [58] with minor modifications. Briefly, Agrobacterium EHA105 carrying Myc-GT-4 and flagTEM2 plasmids were suspended in infiltration buffer to OD600 $=1.0$, except that p19 was diluted to OD600 $=0.6$. For co-infiltration, equal volumes of different Agrobacterium strains were mixed prior to infiltration. The infiltrated parts of leave samples were taken after 48-72 $\mathrm{h}$, and then ground in liquid nitrogen. Nuclei was extracted with Nuclei isolation buffer $(0.25 \mathrm{M}$ sourcose, $15 \mathrm{mM}$ PIPES, pH6.8, $5 \mathrm{mM}$ $\mathrm{MgCl}_{2}, 60 \mathrm{mM} \mathrm{KCl}, 15 \mathrm{mM} \mathrm{NaCl}, 1 \mathrm{mM} \mathrm{CaCl}, 0.9 \%$ Triton X-100, $1 \mathrm{mM}$ PMSF, $2 \mu \mathrm{g} / \mathrm{ml}$ Pepstatin A, $2 \mu \mathrm{g} / \mathrm{ml}$ Aprotinin). Fine powder of each sample were suspended with gentle rotation, then left on ice for $20 \mathrm{~min}$ and filtered by gauze. The colatuies were centrifuged at $11000 \mathrm{~g}$ at $4^{\circ} \mathrm{C}$ for 20 minutes. The sediments were suspended by $400 \mu \mathrm{L}$ IP buffer $(50 \mathrm{mM}$ Tris- $\mathrm{HCl}$, PH7.6, $150 \mathrm{mM} \mathrm{NaCl}, 0.5 \% \mathrm{NP}-40,0.2 \%$ Triton X-100, $2 \mathrm{mM}$ EDTA, and $1 \times$ protease inhibitor cocktail [Roche]). The lysates were left on ice for $2 \mathrm{~h}$ with gentle rotation to make proteins solubilized and centrifuged at $16500 \mathrm{~g}$ at $4^{\circ} \mathrm{C}$ for 30 minutes. Supernatants $(300 \mu \mathrm{L})$ were performed in accordance to the manufacturer's instructions (Thermo Pierce) and incubated with $5 \mu \mathrm{g}$ anti-c-Myc agarose beads for overnight at $4^{\circ} \mathrm{C}$ with slight shaking. To elute the proteins, $25 \mu \mathrm{L} 2 \times$ non-reducing sample buffer were added and heated at $95^{\circ} \mathrm{C}$ for 5 minutes. The presence of the Flag-TEM2 was examined with the anti-Flag antibody (EARTHOX) by western blotting.

\section{Detection of luciferase activity in tobacco leaf}

The assay was performed as described [57]. The 1.5-kb promoter of Cor15A was amplified by PCR using primers (forward: 5'- ATGAAACTGAATAAACTCC CTG-3'; reverse: 5'- GAGAGAGATCTTTAAGATGTG AGA-3') and inserted into pGWB435 to generate promoter::LUC reporter constructs using Gateway system (Invitrogen, USA). The reporter construct $\left(\mathrm{P}_{\text {Cor15A: }}: \mathrm{LUC}\right)$ and the effector constructs (pGWB454, pGWB454-GT-4, pGWB454-TEM2) were transformed into A. tumefaciens strain GV3101 and introduced into tobacco leaves by infiltration. LUC activity was observed with a cold CCD camera (Berthold Technologies, Germany) $2 \mathrm{~d}$ after infiltration.

\section{Statistical analysis}

The data of survival rate in Figure 3e and Figure 6e were subjected to Student's $t$-test analysis using SPSS 11.5 (SPSS Inc., USA).

\section{Availability of supporting data}

The data sets supporting the results of this article are included within the article and its additional files.

\section{Additional files}

Additional file 1: Auto-activity of BD-GT-4, BD-GT-4N, BD-GT-4C, AD-GT-4 and AD-TEM2 in the yeast assay.

Additional file 2: Negative control of bimolecular fluorescence complementation (BiFC) assay.

Additional file 3: Primers used for Q-PCR.

\section{Competing interests}

The authors declare that they have no competing interests.

\section{Authors' contributions}

XHW and QTL conducted experiments and drafted the initial manuscript. HWC, WKZ, BM contributed to data analysis. JSZ and SYC conceived of the study, obtained funding, analyzed data and finished the final manuscript. All authors read and approved the final manuscript.

\section{Acknowledgements}

This work is supported by the National Key Basic Research Project of China (2012CB1 14202), the CAS project (KSCX3-EW-N-07) and the National Transgenic Research Program (2014ZX0800926B; 2014ZX08004003-005).

Received: 16 June 2014 Accepted: 18 November 2014

Published online: 03 December 2014

\section{References}

1. Hao YJ, Wei W, Song QX, Chen HW, Zhang YQ, Wang F, Zou HF, Lei G, Tian AG, Zhang WK, Ma B, Zhang JS, Chen SY: Soybean NAC transcription factors promote abiotic stress tolerance and lateral root formation in transgenic plants. Plant J 2011, 68(2):302-313.

2. He XJ, Mu RL, Cao WH, Zhang ZG, Zhang JS, Chen SY: AtNAC2, a transcription factor downstream of ethylene and auxin signaling pathways, is involved in salt stress response and lateral root development. Plant J 2005, 44(6):903-916.

3. Hu H, Dai M, Yao J, Xiao B, Li X, Zhang Q, Xiong L: Overexpressing a NAM, ATAF, and CUC (NAC) transcription factor enhances drought resistance and salt tolerance in rice. Proc Natl Acad Sci U S A 2006, 103(35):12987-12992.

4. Hu H, You J, Fang Y, Zhu X, Qi Z, Xiong L: Characterization of transcription factor gene SNAC2 conferring cold and salt tolerance in rice. Plant $\mathrm{Mol}$ Biol 2008, 67(1-2):169-181.

5. Jiang $Y$, Deyholos MK: Functional characterization of Arabidopsis $\mathrm{NaCl}$-inducible WRKY25 and WRKY33 transcription factors in abiotic stresses. Plant Mol Biol 2009, 69(1-2):91-105.

6. Lee S, Seo PJ, Lee HJ, Park CM: A NAC transcription factor NTL4 promotes reactive oxygen species production during drought-induced leaf senescence in Arabidopsis. Plant J 2012, 70(5):831-844.

7. Liao Y, Zou HF, Wang HW, Zhang WK, Ma B, Zhang JS, Chen SY: Soybean GmMYB76, GmMYB92, and GmMYB177 genes confer stress tolerance in transgenic Arabidopsis plants. Cell Res 2008, 18(10):1047-1060.

8. Liao Y, Zou HF, Wei W, Hao YJ, Tian AG, Huang J, Liu YF, Zhang JS, Chen SY: Soybean GmbZIP44, GmbZIP62 and GmbZIP78 genes function as negative regulator of $A B A$ signaling and confer salt and freezing tolerance in transgenic Arabidopsis. Planta 2008, 228(2):225-240.

9. Niu CF, Wei W, Zhou QY, Tian AG, Hao YJ, Zhang WK, Ma B, Lin Q, Zhang ZB, Zhang JS, Chen SY: Wheat WRKY genes TaWRKY2 and TaWRKY19 regulate abiotic stress tolerance in transgenic Arabidopsis plants. Plant Cell Environ 2012, 35(6):1156-1170.

10. Dehesh K, Hung H, Tepperman JM, Quail PH: GT-2: a transcription factor with twin autonomous DNA-binding domains of closely related but different target sequence specificity. EMBO J 1992, 11(11):4131-4144.

11. Zhou DX: Regulatory mechanism of plant gene transcription by GT-elements and GT-factors. Trends Plant Sci 1999, 4(6):210-214. 
12. Nagano Y: Several features of the GT-factor trihelix domain resemble those of the Myb DNA-binding domain. Plant Physiol 2000, 124(2):491-494.

13. Riechmann JL, Heard J, Martin G, Reuber L, Jiang C, Keddie J, Adam L, Pineda O, Ratcliffe OJ, Samaha RR, Creelman R, Pilgrim M, Broun P, Zhang JZ, Ghandehari D, Sherman BK, Yu G: Arabidopsis transcription factors: genome-wide comparative analysis among eukaryotes. Science 2000, 290(5499):2105-2110.

14. Gao MJ, Lydiate DJ, Li X, Lui H, Gjetvaj B, Hegedus DD, Rozwadowski K: Repression of seed maturation genes by a trihelix transcriptional repressor in Arabidopsis seedlings. Plant Cell 2009, 21(1):54-71.

15. Kaplan-Levy RN, Brewer PB, Quon T, Smyth DR: The trihelix family of transcription factors-light, stress and development. Trends Plant Sci 2012, 17(3):163-171

16. Fluhr R, Kuhlemeier C, Nagy F, Chua NH: Organ-specific and light-induced expression of plant genes. Science 1986, 232(4754):1106-1112.

17. Green PJ, Kay SA, Chua NH: Sequence-specific interactions of a pea nuclear factor with light-responsive elements upstream of the rbcS-3A gene. EMBO J 1987, 6(9):2543-2549.

18. Zhou DX, Li YF, Rocipon M, Mache R: Sequence-specific interaction between S1F, a spinach nuclear factor, and a negative cis-element conserved in plastid-related genes. J Biol Chem 1992, 267(33):23515-23519.

19. Villain P, Clabault G, Mache R, Zhou DX: S1F binding site is related to but different from the light-responsive GT-1 binding site and differentially represses the spinach rps1 promoter in transgenic tobacco. $J$ Biol Chem 1994, 269(24):16626-16630.

20. Buchel AS, Molenkamp R, Bol JF, Linthorst HJ: The PR-1a promoter contains a number of elements that bind GT-1-like nuclear factors with different affinity. Plant Mol Biol 1996, 30(3):493-504.

21. Park HC, Kim ML, Kang YH, Jeon JM, Yoo JH, Kim MC, Park CY, Jeong JC, Moon BC, Lee JH, Yoon HW, Lee SH, Chung WS, Lim CO, Lee SY, Hong JC, Cho MJ: Pathogen- and NaCl-induced expression of the SCaM-4 promoter is mediated in part by a GT-1 box that interacts with a GT-1-like transcription factor. Plant Physiol 2004, 135(4):2150-2161.

22. Green PJ, Yong MH, Cuozzo M, Kano-Murakami $Y$, Silverstein $P$, Chua NH: Binding site requirements for pea nuclear protein factor GT-1 correlate with sequences required for light-dependent transcriptional activation of the rbcS-3A gene. EMBO J 1988, 7(13):4035-4044.

23. Dehesh K, Bruce WB, Quail PH: A trans-acting factor that binds to a GT-motif in a phytochrome gene promoter. Science 1990, 250(4986):1397-1399.

24. Perisic O, Lam E: A tobacco DNA binding protein that interacts with a light-responsive box II element. Plant Cell 1992, 4(7):831-838.

25. Brewer PB, Howles PA, Dorian K, Griffith ME, Ishida T, Kaplan-Levy RN, Kilinc A, Smyth DR: PETAL LOSS, a trihelix transcription factor gene, regulates perianth architecture in the Arabidopsis flower. Development 2004, 131(16):4035-4045.

26. Lin Z, Griffith ME, Li X, Zhu Z, Tan L, Fu Y, Zhang W, Wang X, Xie D, Sun C: Origin of seed shattering in rice (Oryza sativa L.). Planta 2007 226(1):11-20.

27. Li X, Qin G, Chen Z, Gu H, Qu L: A gain-of-function mutation of transcriptional factor PTL results in curly leaves, dwarfism and male sterility by affecting auxin homeostasis. Plant Mol Biol 2008, 66(3):315-327.

28. Lampugnani ER, Kilinc A, Smyth DR: PETAL LOSS is a boundary gene that inhibits growth between developing sepals in Arabidopsis thaliana. Plant J 2012, 71(5):724-735.

29. Xie ZM, Zou HF, Lei G, Wei W, Zhou QY, Niu CF, Liao Y, Tian AG, Ma B, Zhang WK, Zhang JS, Chen SY: Soybean Trihelix transcription factors GmGT-2A and GmGT-2B improve plant tolerance to abiotic stresses in transgenic Arabidopsis. PLoS One 2009, 4(9):e6898.

30. Fang Y, Xie K, Hou X, Hu H, Xiong L: Systematic analysis of GT factor family of rice reveals a novel subfamily involved in stress responses. Mol Genet Genomics 2010, 283(2):157-169.

31. Yoo CY, Pence HE, Jin JB, Miura K, Gosney MJ, Hasegawa PM, Mickelbart MV: The Arabidopsis GTL1 transcription factor regulates water use efficiency and drought tolerance by modulating stomatal density via transrepression of SDD1. Plant Cell 2010, 22(12):4128-4141.

32. Weng H, Yoo CY, Gosney MJ, Hasegawa PM, Mickelbart MV: Poplar GTL1 Is a Ca2+/Calmodulin-Binding Transcription Factor that Functions in Plant Water Use Efficiency and Drought Tolerance. PLoS One 2012, 7(3):e32925.

33. Li Q, Liu J, Tan D, Allan AC, Jiang Y, Xu X, Han Z, Kong J: A genome-wide expression profile of salt-responsive genes in the apple rootstock Malus zumi. Int J Mol Sci 2013, 14(10):21053-21070.
34. Griffith ME, da Silva CA, Smyth DR: PETAL LOSS gene regulates initiation and orientation of second whorl organs in the Arabidopsis flower. Development 1999, 126(24):5635-5644

35. Wang XH: Functional Analysis of Arabidopsis Trihelix Transcription Factors Under Salt Stress. PhD Thesis. Beijing 100101, China: Institute of Genetics and Developmental Biology, Chinese Academy of Sciences; 2013.

36. Kuhn RM, Caspar T, Dehesh K, Quail PH: DNA binding factor GT-2 from Arabidopsis. Plant Mol Biol 1993, 23(2):337-348.

37. Ayadi M, Delaporte V, Li YF, Zhou DX: Analysis of GT-3a identifies a distinct subgroup of trihelix DNA-binding transcription factors in Arabidopsis. FEBS Lett 2004, 562(1-3):147-154.

38. Rushton PJ, Somssich IE, Ringler P, Shen QJ: WRKY transcription factors. Trends Plant Sci 2010, 15(5):247-258

39. Castillejo C, Pelaz S: The balance between CONSTANS and TEMPRANILLO activities determines FT expression to trigger flowering. Curr Biol 2008, 18(17):1338-1343.

40. Xi J, Qiu Y, Du L, Poovaiah BW: Plant-specific trihelix transcription factor AtGT2L interacts with calcium/calmodulin and responds to cold and salt stresses. Plant Sci 2012, 185-186:274-280.

41. Murata J, Takase H, Hiratsuka K: Characterization of a novel GT-box binding protein from Arabidopsis. Plant Bioltechnol 2002, 19:103-112

42. Wang MY, Zhao PM, Cheng HQ, Han LB, Wu XM, Gao P, Wang HY, Yang CL, Zhong NQ, Zuo JR, Xia GX: The cotton transcription factor TCP14 functions in auxin-mediated epidermal cell differentiation and elongation. Plant Physiol 2013, 162(3):1669-1680.

43. Ni M, Dehesh K, Tepperman JM, Quail PH: GT-2: in vivo transcriptional activation activity and definition of novel twin DNA binding domains with reciprocal target sequence selectivity. Plant Cell 1996, 8(6):1041-1059.

44. Le Gourrierec J, Li YF, Zhou DX: Transcriptional activation by Arabidopsis GT-1 may be through interaction with TFIIA-TBP-TATA complex. Plant J 1999, 18(6):663-668.

45. Lin C, Thomashow MF: A cold-regulated Arabidopsis gene encodes a polypeptide having potent cryoprotective activity. Biochem Biophys Res Commun 1992, 183(3):1103-1108.

46. Nakayama K, Okawa K, Kakizaki T, Inaba T: Evaluation of the protective activities of a late embryogenesis abundant (LEA) related protein, Cor15am, during various stresses in vitro. Biosci Biotechnol Biochem 2008, 72(6):1642-1645.

47. Artus NN, Uemura M, Steponkus PL, Gilmour SJ, Lin C, Thomashow MF: Constitutive expression of the cold-regulated Arabidopsis thaliana COR15a gene affects both chloroplast and protoplast freezing tolerance. Proc Natl Acad Sci U S A 1996, 93(23):13404-13409.

48. Song C, Je J, Hong JK, Lim CO: Ectopic expression of an Arabidopsis dehydration-responsive element-binding factor DREB2C improves salt stress tolerance in crucifers. Plant Cell Rep 2014, 33(8):1239-1254.

49. Dehesh K, Smith LG, Tepperman JM, Quail PH: Twin autonomous bipartite nuclear localization signals direct nuclear import of GT-2. Plant J 1995, 8(1):25-36.

50. Hiratsuka K, Wu X, Fukuzawa H, Chua NH: Molecular dissection of GT-1 from Arabidopsis. Plant Cell 1994, 6(12):1805-1813.

51. Kagaya $Y$, Hattori T: Arabidopsis transcription factors, RAV1 and RAV2, are regulated by touch-related stimuli in a dose-dependent and biphasic manner. Genes Genet Syst 2009, 84(1):95-99.

52. Guo ZJ, Chen XJ, Wu XL, Ling JQ, Xu P: Overexpression of the AP2/EREBP transcription factor OPBP1 enhances disease resistance and salt tolerance in tobacco. Plant Mol Biol 2004, 55(4):607-618.

53. Zhang G, Chen M, Li L, Xu Z, Chen X, Guo J, Ma Y: Overexpression of the soybean GmERF3 gene, an AP2/ERF type transcription factor for increased tolerances to salt, drought, and diseases in transgenic tobacco. J Exp Bot 2009, 60(13):3781-3796.

54. Zhu Q, Zhang J, Gao X, Tong J, Xiao L, Li W, Zhang H: The Arabidopsis AP2/ERF transcription factor RAP2.6 participates in ABA, salt and osmotic stress responses. Gene 2010, 457(1-2):1-12.

55. Wittwer CT, Ririe KM, Andrew RV, David DA, Gundry RA, Balis UJ: The LightCycler: a microvolume multisample fluorimeter with rapid temperature control. Biotechniques 1997, 22(1):176-181.

56. Hao YJ, Song QX, Chen HW, Zou HF, Wei W, Kang XS, Ma B, Zhang WK, Zhang JS, Chen SY: Plant NAC-type transcription factor proteins contain a NARD domain for repression of transcriptional activation. Planta 2010, 232(5):1033-1043. 
57. Song QX, Li QT, Liu YF, Zhang FX, Ma B, Zhang WK, Man WQ, Du WG, Wang GD, Chen SY, Zhang JS: Soybean GmbZIP123 gene enhances lipid content in the seeds of transgenic Arabidopsis plants. J Exp Bot 2013, 64(14):4329-4341.

58. Liu L, Zhang Y, Tang S, Zhao Q, Zhang Z, Zhang H, Dong L, Guo H, Xie Q: An efficient system to detect protein ubiquitination by agroinfiltration in Nicotiana benthamiana. Plant J 2010, 61(5):893-903.

doi:10.1186/s12870-014-0339-7

Cite this article as: Wang et al.: Trihelix transcription factor GT-4

mediates salt tolerance via interaction with TEM2 in Arabidopsis. BMC

Plant Biology 2014 14:339.

\section{Submit your next manuscript to BioMed Central and take full advantage of:}

- Convenient online submission

- Thorough peer review

- No space constraints or color figure charges

- Immediate publication on acceptance

- Inclusion in PubMed, CAS, Scopus and Google Scholar

- Research which is freely available for redistribution 